\title{
Design and Simulation of Stratospheric Airship Energy Management System
}

\author{
Kangwen Sun ${ }^{1, a}$, Dongdong $\mathrm{Xu}^{2, \mathrm{~b}}$ and Xinyun Zhang ${ }^{3, \mathrm{c}}$ \\ ${ }^{1-3}$ School of Aeronautic Science and Engineering, Beijing University of Aeronautics and Astronautics, \\ Key Laboratory of Aircraft Advanced Design Technology Ministry of Industry and Information \\ Technology, Beijing 100191, China

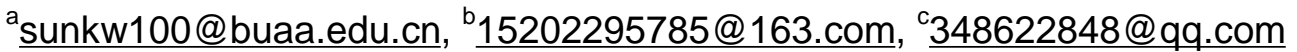

Keywords: Stratospheric airship; Energy management; DC-DC converter; Bus voltage; Fault isolation.

Abstract. Redundancy design scheme and control strategy of the energy management system for a stratospheric airship are proposed, which use the coordination control between the photovoltaic cell and lithium battery to maintain the DC bus voltage constant, so as to guarantee the stability of the motor load. The system can switch to corresponding working state according to different conditions through the analysis of the different control modes of unidirectional DC-DC and bidirectional DC-DC. The paper used MATLAB simulation, through increasing or decreasing the load at DC bus terminals and changing the input energy to detect the stability and reliability of the system. At the same time, the reconstruction ability of the system is tested by setting fault information for key nodes. The simulation results show that the proposed design scheme and control strategy not only maintains the stability of the bus voltage, but also makes fault isolated and achieves system reconstruction and effectively protects the storage battery.

\section{Introduction}

In recent years, the unique advantage of space resources and solar energy in near space has attracted focus worldwide. Currently, America, Japan, some European countries and other developed nations are taking advantage of their mastered advanced technology to carrying out a series of research and verification tests of stratospheric airship, expecting to quickly occupy the outpost of near space-stratosphere[1,2].

Different from the traditional airship, stratospheric airship has superiority of long endurance, wide coverage, strong survival ability, low cost of manufacturing and maintenance, timeliness and accuracy in wireless communication and other features [3], making it high value in military and civilian and a perfect flying platform for communications relay, remote alarm, high precision earth observation, environmental monitoring, directing rescue and other missions [4].

Therefore, based on solar cell - Lithium battery hybrid energy systems [5], an energy management system (EMS) design program of stratospheric airship was proposed, which can make bad key nodes isolated and achieve system reconstruction and also can switch freely between different operating modes. System structure is shown in Figure 1. EMS can be reliable through reasonable control model design and accurate modeling and simulation [6].

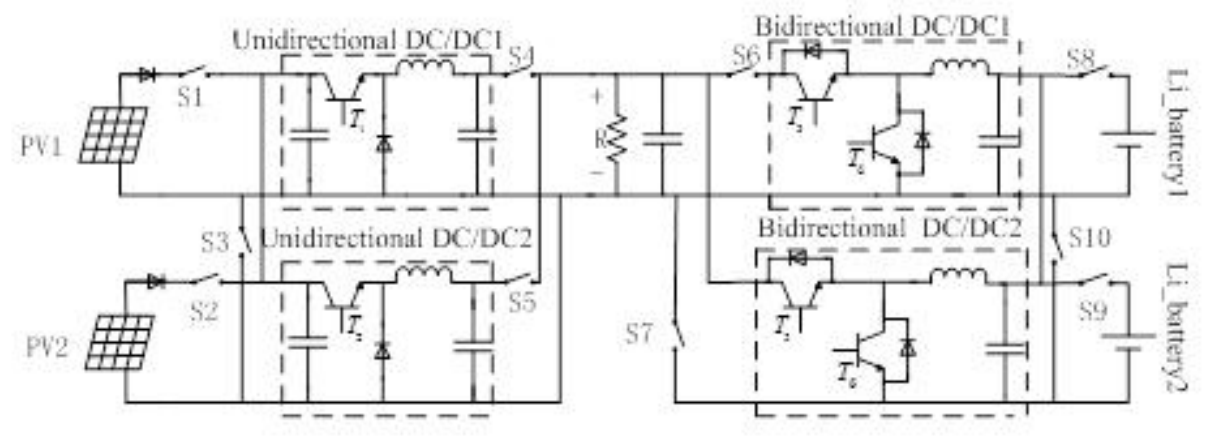

Fig. 1 The structure of system 
Different operating modes of the system. The system mainly consists of photovoltaic cells 1 (PV1), photovoltaic cells 2 (PV2), unidirectional DC/DC converter 1(UDC1), unidirectional DC/DC converter 2(UDC2), bidirectional DC/DC converter 1(BDC1), bidirectional DC/DC converter 2(BDC2), lithium battery 1, lithium battery 2 and power switch 1 to 10 . PV, DC converters, and lithium battery are considered as key nodes in recycling energy systems consisting of PV and lithium battery. Status of key nodes have an important effect on the entire EMS. For stratospheric airship, redundant design of key nodes can make their fault isolated and achieve system reconstruction, which maintain energy system stability as much as possible.

Key nodes are normal. When all key nodes are working properly, in addition to the switch 3 and the switch 10 are off, the other switches are in the closed state. In this case, PV1 and PV2 are in parallel with bus bar and supply power to the load (motor) after being connected to UDC1 and UDC2 respectively. At the same time, lithium battery 1 and lithium battery 2 are also in parallel with the bus bar after being connected to BDC1 and BDC2 respectively.

When key nodes are working properly, the system can achieve composite maximum power point tracking (MPPT) [7]. Each PV array is equipped with independent MPPT controller to track peak power, thereby avoiding mutual influence of power output between different solar array areas caused by hull surfaces [8]. In this case, the working situation of system can be divided into three operation modes based on the value of the irradiation, lithium battery capacity (SOC), output power of PV $\left(P_{p v}\right)$, and power consumption of load $\left(P_{\text {load }}\right)$.

Mode 1. When irradiation is sufficient, $P_{p v}>P_{\text {load }}$, and SOC $<95 \%$, each UDC works in constant voltage $(\mathrm{CV})$ mode to get a stable voltage for DC bus and each bidirectional DC works in buck mode to charge lithium battery. That means solar cell can provide more power than load needed, solar cell powers load and residual power charges battery synchronously. In addition, the value of charging current of lithium battery can be changed by setting reference current in buck control circuit, which can not only achieve the maximum use of solar energy but also protect the lithium battery. If SOC is more than 95\%, two BDCs stop working and the system is powered by solar energy alone.

Mode 2. When either irradiation is insufficient or load power demand increases suddenly in mode 1, namely $P_{p v}<P_{\text {load }}$, the operation mode of UDC must immediately change from CV to MPPT mode. At the same time, BDC change from buck to boost mode to keep bus voltage stable. Solar cell cannot provide enough energy to power load, the shortage will be complemented by battery via the BDC.

Mode 3. At night, UDC stop working. Lithium batteries working in boost mode power load alone and bus voltage is kept stable by BDC.

Key nodes have problem. If a UDC does not work properly, it should be isolated and energy system should be reconstructed to maintain working properly. UDC1 fails, for example, break switch 4 to isolate bad UDC1 and connect switch 3 based on normal situation. That means output of PV1 and PV2 are in parallel and power conversion is achieved by UDC2 alone.

If a bidirectional DC / DC fails, the same concept of sharing system components is used to achieve fault isolation and functional remodeling. BDC1 fails, for example, break switch 6 to isolate bad BDC1 and connect switch 10 based on normal situation. That means lithium battery1 and lithium battery 2 are in parallel and power conversion is achieved by BDC2 alone.

If PV1 fails, for example, switch 1 should be disconnected to isolate bad PV1. If lithium battery1 fails, for example, switch 8 should be disconnected. Whether it is PV or lithium battery fails, energy system of stratospheric airship is not complete, which makes pre-designed system no longer meet energy needs for the circadian cycle work. In this case, we need to change the mission profile of the airship, such as reducing working hours of propulsion motor, making it match the current energy supply ability after fault isolation.

System control circuit. The above shows UDC can work between MPPT and CV mode freely. BDC can work between buck and boost mode freely [12].

MPPT mode of UDC. Currently, algorithm of tracking solar array peak power mainly comprises constant voltage method, perturbation and observation method, the incremental conductance method 
[9]. Perturbation and observation is the most commonly used method in photovoltaic system though it cannot track fast enough when radiation is in dramatic changes [10]. However, taking the actual situation into account of that attitude angle of stratospheric airship changes slowly, this method can be used in this paper.

CV mode of UDC. UDC works in CV mode to control DC bus voltage to be a stable value. The control block diagram of CV mode is shown in Figure 2 [11].

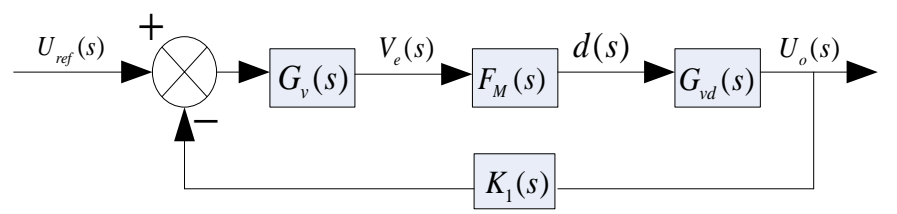

Fig. 2 Control block diagram of UDC working in constant voltage mode

Where, $U_{r e f}(s), V_{e}(s), d(s), F_{M}(s), G_{v d}(s), G_{v}(s), K_{1}(s)$ are reference voltage of DC bus, control voltage, duty cycle, the transfer function from pulse width modulator to duty cycle, the transfer function from duty cycle to output, voltage controller and sampling network transfer function respectively.

Buck mode of BDC. BDC in buck mode is equivalent to a charger charging lithium batteries. Chargers must contain the CV control circuit and constant current (CC) control loop. The strategy of dual closed loop voltage and current PI controllers is adopted in this paper as shown in Figure 3 [13].

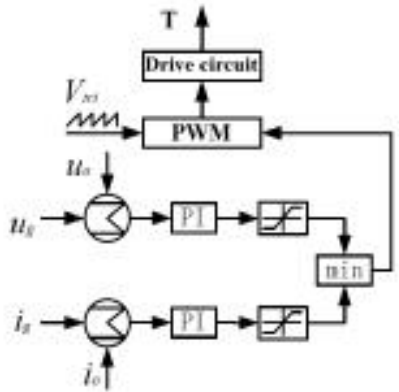

Fig. 3 Control diagram of BDC working in buck mode

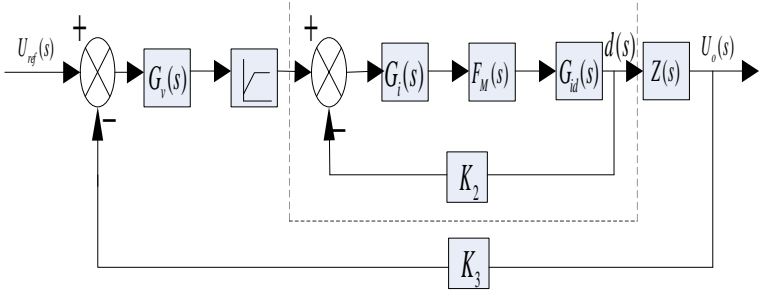

Fig. 4 Control diagram of BDC working in Boost mode $u_{g}$ and $i_{g}$ are the feedback voltage and current of buck circuit respectively. $u_{o}$ and $i_{o}$ are the target voltage and current of buck circuit respectively. $V_{t r i}$ is excitation voltage generated by the oscillator. $T$ is the controlled power switch when BDC works in buck mode.

As shown in figure 3, control circuit consists of a constant voltage PI controller and a constant current PI controller. By comparing the size of error signals which are the difference between target voltage/current and feedback voltage/current, the smaller one will be the PWM control signal of the closed loop circuit. In CV mode, the output voltage of the converter is controlled by the target voltage and the voltage difference of the voltage loop is approximately equal to zero while the current difference of current loop is relatively large [14].

Boost mode of BDC. BDC works in boost mode when lithium battery powers load alone or with PV together. In this case, lithium battery discharge in a way of constant pressure limiting to protect itself. The control block diagram of boost mode is shown in Figure 4 [15].

Where, $Z(s), K_{2}(s), K_{3}(s)$ are load transfer function and current and voltage sampling network transfer function respectively. Dashed box is inner current loop discharging in limiting way. The outer loop ensures DC bus voltage stable.

System modeling. According to the above, components consisting of energy system should be modeled and controller for DC controller should be designed. In this paper, MATLAB/Simulink is used to design and analysis controller and model each component.

Lithium battery modeling. Tremblay [16] etc. use polarization voltage, not polarization resistance to correct the former equation, which eliminate the algebraic loop problem in simulation. So, the battery voltage in discharge model can be obtained as: 


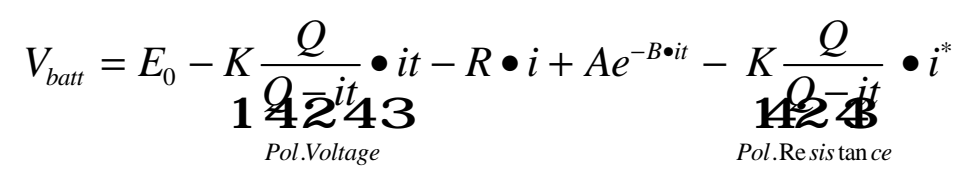

Where: $V_{\text {batt }}=$ battery voltage $(V) ; E_{0}=$ battery constant voltage $(V) ; K=$ polarization constant $(V /(A h))$ or polarization resistance $(\Omega) ; Q=$ battery capacity $(A h) ; i t=\int i \mathrm{dt}=$ actual battery charge $(A h) ; A=$ exponential zone amplitude $(V) ; B=$ exponential zone time constant inverse $(A h)^{-1} ; R=$ internal resistance $(\Omega) ; i=$ battery current $(A) ; i^{*}=$ filtered current $(A)$.

Similar to the discharge model, the complete model for charging is:

$$
V_{b c t t}=E_{0}-R \bullet i-K \frac{Q}{i t-0.1 \bullet Q} \bullet i^{*}-K \frac{Q}{Q-i t} \bullet i t+A e^{-B \cdot i t}
$$

According to above formula and specific parameters, simulation model shown in Figure 5 is created.

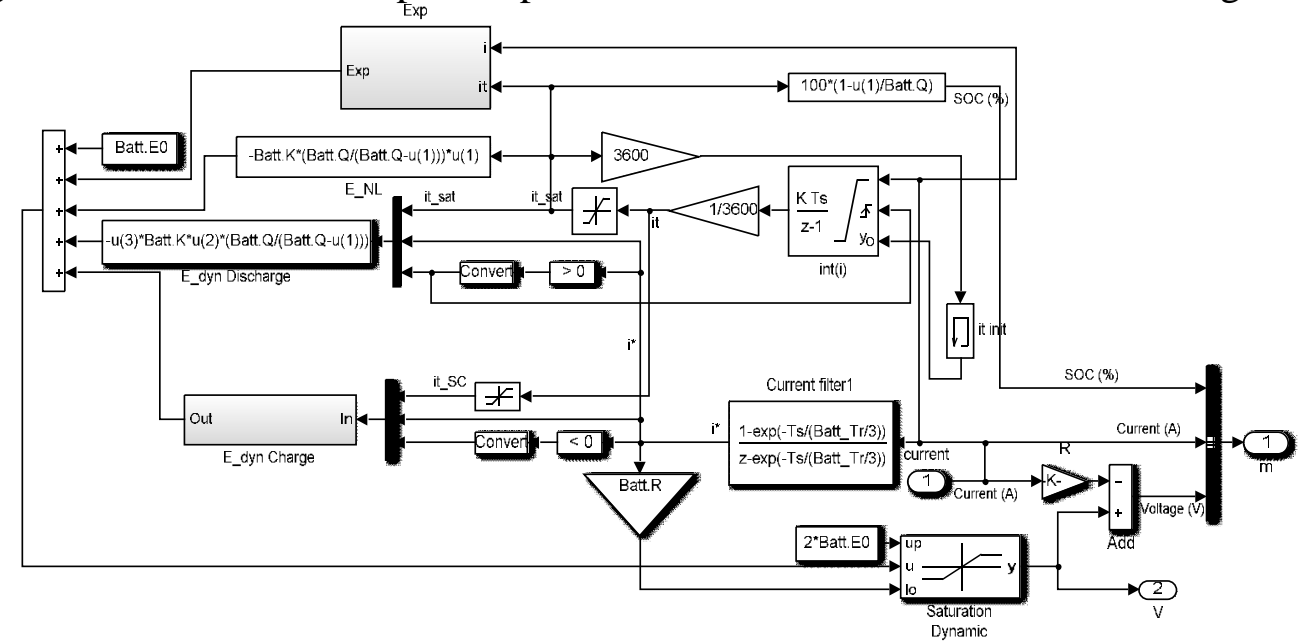

Fig. 5 Lithium battery simulation model

PV modeling. PV equivalent circuit model is shown in Figure 6 [17]. The voltage-current characteristic equation of a solar cell can be given as below:

$$
I=I_{p h}-I_{0} e^{\frac{Q\left(I R_{s}+V\right) Q_{i}}{k T}-1}-\frac{V+I R_{s}}{R_{s h}}
$$

Where, $I_{p h}$ is the light-generated current. $R_{s h}$ is the internal shunt resistance and $R_{s}$ is the series resistor. $Q_{d}(=1$ or 2$)$ is the diode quality factor. $k\left(=1.38 \times 10^{-23} \mathrm{~J} / \mathrm{K}\right)$ is a Boltzmann's constant. $T(=\mathrm{t}+273 \mathrm{~K})$ is the cell's working temperature, $q\left(=1.6 \times 10^{-19} \mathrm{C}\right)$ is an electron charge. According to the above mathematical expression, PV simulation model shown in Figure 7 can be created.

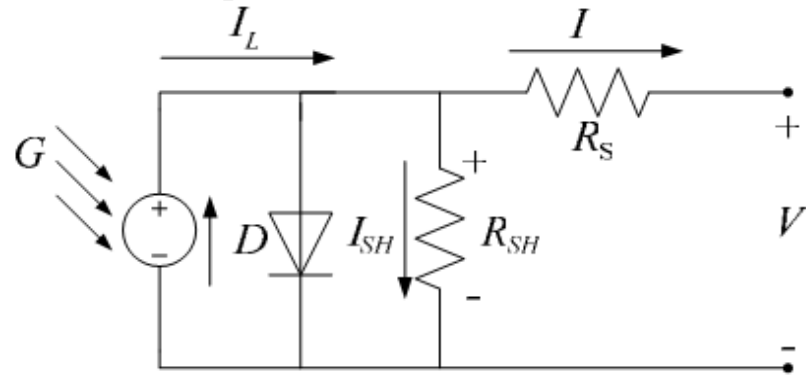

Fig. 6 Circuit diagram of the PV model

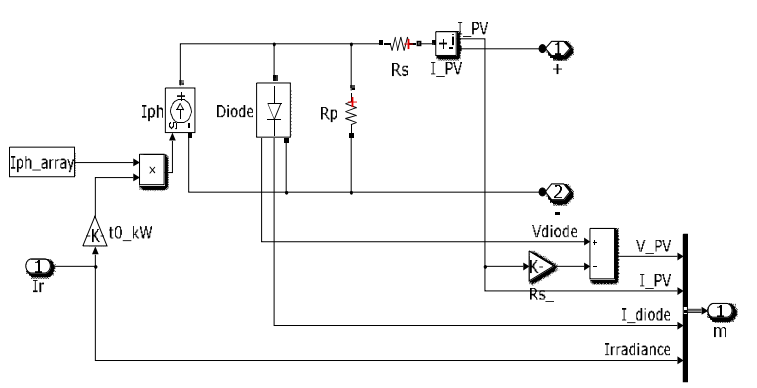

Fig. 7 Solar cell simulation model

Motor (load) modeling. The brushless DC motor (BDCM) is often used as stratospheric airship propulsion device because of its many advantages like small size, light weight, high efficiency, low inertia, high precision control and so on [18]. BDCM simulation model is established by referring [18]. The load power can be obtained by setting different speed and torque as the input of the model. 
DC converter modeling. DC converter undertakes power conversion in energy system. At same time, system control strategy is performed based on DC converter. In this paper, the MOSFET in Simulink is selected as power switch T, switching frequency of which is set to $25 \mathrm{kHz}$.

The inductance and capacitance in DC simulation model play roles of filtering one hand, on the other hand, capacitance acts as a decoupling device in cascade circuit of two stages, avoiding system instability because of the resonance [19]. UDC simulation model is shown in Figure 8 and Figure 9 is BDC.

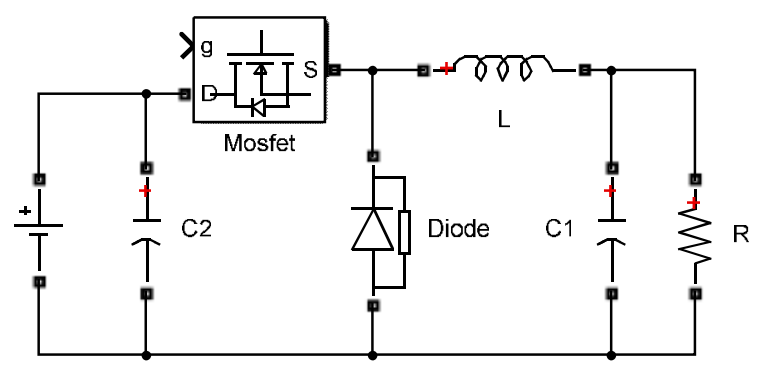

Fig. 8 Unidirectional DC-DC simulation model

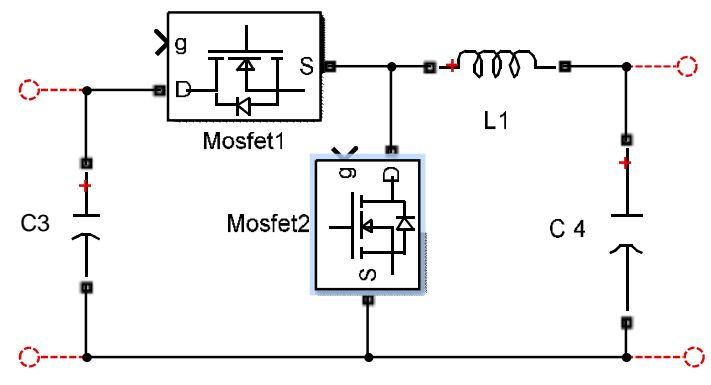

Fig. 9 Bidirectional DC-DC simulation model

Simulation results. In order to verify reliability of the proposed energy system and correctness of the control strategy, models above should be combined into an overall Simulink simulation model. According to the proposed control strategy in this paper, we need to build solar energy harvesting and load consumption module and fault detection module. To simplify the complexity of the simulation model, the paper gives the value of estimation for solar energy harvesting $P_{p v}$ by giving irradiation. The value of energy consumption $P_{\text {load }}$ is given by setting the speed and torque in motor model. The ability of fault isolation and system reconstruction is tested by setting the different state of switch series at different time point.Simulation parameters are listed as follows:

PV1 and PV2: peak power 1240W (standard state), output voltage 150-300V;

Lithium battery1 and lithium battery2: rated voltage $45 \mathrm{~V}$, capacity $150 \mathrm{Ah}$, overcharge current $25 \mathrm{~A}$; over-discharge current $40 \mathrm{~A}$, over-charge voltage $53 \mathrm{~V}$, over-discharge voltage $41 \mathrm{~V}$;

UDC: input voltage $150-300 \mathrm{~V}$, output voltage $100 \mathrm{~V}$;

BDC: output voltage in boost mode $100 \mathrm{~V}$, output voltage in buck mode $56 \mathrm{~V}$;

Load (motor): rated power $1050 \mathrm{~W}$, rated voltage $100 \mathrm{~V}$.

Figure 10 shows the PV voltage $V_{p v 1}$ and $V_{p v 2}$, DC bus voltage $V_{b u s}$, the lithium battery charge/discharge current $I_{L i}$, output power of PV $P_{p v}$ and the BDCM speed of the simulation results. Simulation parameters are described below. The reference charging current Iref of BDC working in buck mode is $12 \mathrm{~A}$. The motor reference speed and torque are $1050 \mathrm{rad} / \mathrm{s}$ and $2.1 \mathrm{~N} / \mathrm{m}$ respectively before $0.2 \mathrm{~s}$ and torque increased by 5 times at $0.2 \mathrm{~s}$, which is equivalent to power consumption increasing 5 times. Irradiance of PV1 and PV2 are respectively $980 \mathrm{~W} / \mathrm{m}^{2}$ and $950 \mathrm{~W} / \mathrm{m}^{2}$ before $0.5 \mathrm{~s}$ and mutate to $290 \mathrm{~W} / \mathrm{m}^{2}$ and $300 \mathrm{~W} / \mathrm{m}^{2}$ at $0.5 \mathrm{~s}$, but they restore to $980 \mathrm{~W} / \mathrm{m}^{2}$ and $950 \mathrm{~W} / \mathrm{m}^{2}$ respectively after $0.8 \mathrm{~s}$. The initial SOC of the lithium battery is $83.2 \%$. Key nodes have no fault initially, namely, the state of switch sequence 1 to 10 is $\left[\begin{array}{lllllllll}1 & 1 & 0 & 1 & 1 & 1 & 1 & 1 & 1\end{array}\right]$ (1 means closed, 0 off).

The reliability of stratospheric airship energy systems proposed in this article can be tested by setting imbalance between energy supply and demand above.

The simulation results can be seen from Figure 10. Before $0.5 \mathrm{~s}$ irradiation is sufficient and system determines $P_{p v}>P_{\text {load }}$, then UDC works in CV mode to keep bus voltage $100 \mathrm{~V}$ and BDC works in buck to charge lithium battery according to Iref . It can be included that system has the capability to respond quickly to reference bus voltage and BDCM speed though load power increasing 5 times at $0.2 \mathrm{~s}$. At $0.5 \mathrm{~s}$, the system determines $P_{p v}<P_{\text {load }}$ due to the weak irradiation, then UDC switches to MPPT mode and BDC works in boost mode powering load with PV together. Although there have some fluctuations in bus voltage and motor speed when work modes make 
changes, they soon returned to normality and continue to maintain stable operation. At $0.8 \mathrm{~s}$, system changes operation mode again due to change of the irradiation. However, this time there have no fluctuations in bus voltage and speed compared with the situation in $0.5 \mathrm{~s}$, the reason of which is that dynamic characteristics of the PV is better than lithium batteries [20]. PV and lithium battery power load together from $0.5 \mathrm{~s}$ to $0.8 \mathrm{~s}$ and PV powers load alone after $0.8 \mathrm{~s}$.
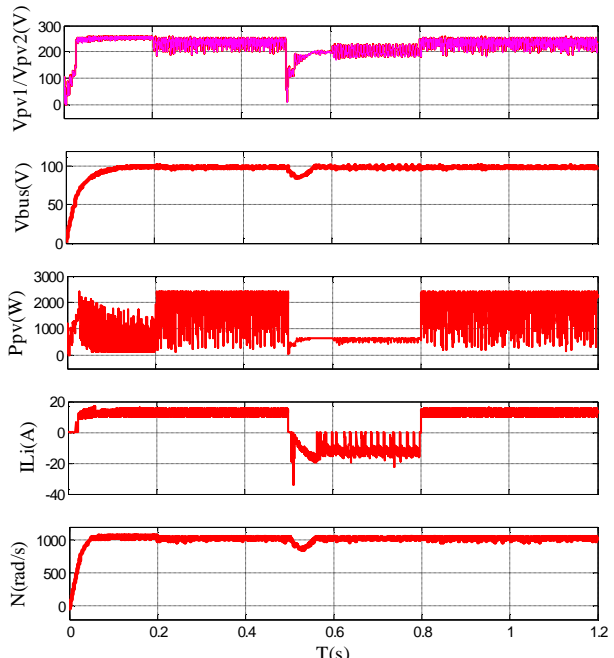

Fig.10 Simulation waveforms with system working in different modes ( Iref $=12 \mathrm{~A}$ )
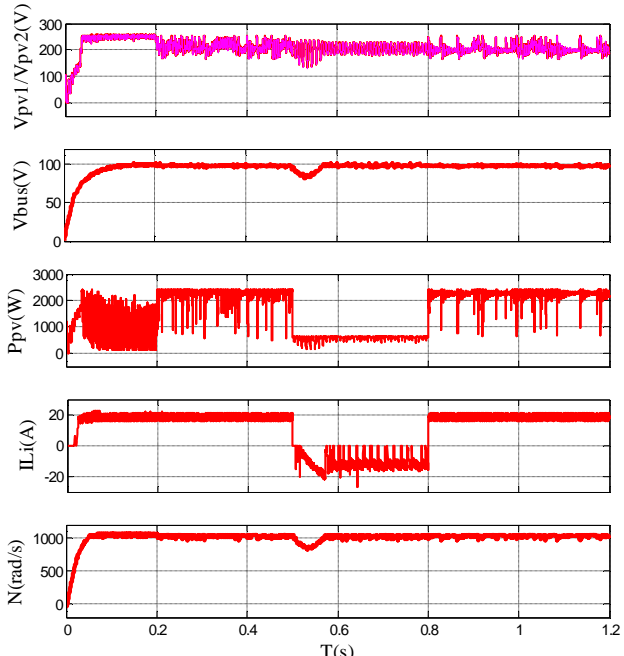

Fig. 11 Simulation waveforms with Iref =20A compared with figure 10

Simulation results above show that the proposed stratospheric airship energy system has better coordination. Figure 11 is the result when Iref is set to 20 A compared with figure 10 . It can be seen that the system not only can maintain stable operation, but also maximize the use of current solar energy by setting different reference current.
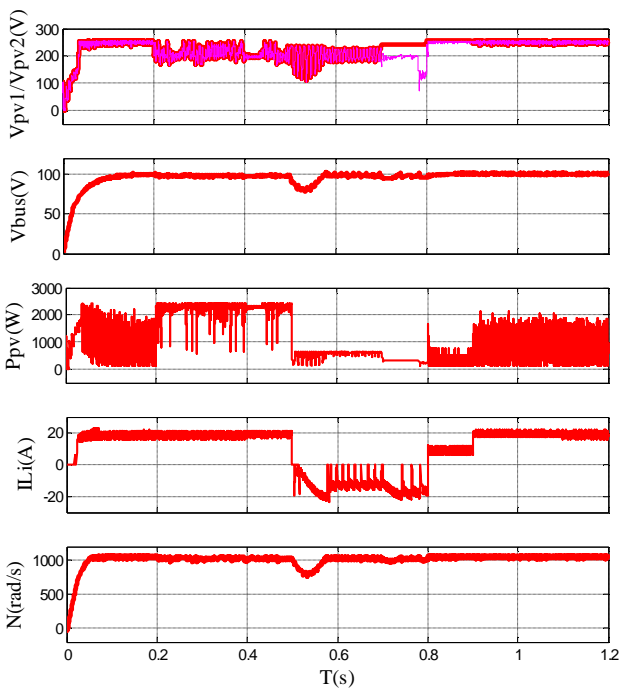

Fig. 12 Simulation waveform with fault isolation and system reconstruction

Figure 12 is a simulation result of fault isolation and reconfiguration based on figure 13. The state of

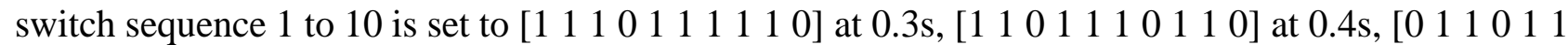

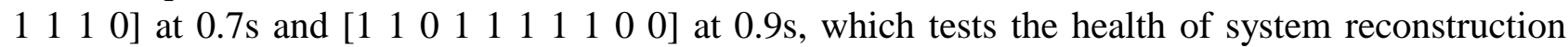
corresponding to UDC1 failing, BDC2 failing, PV1 failing and lithium battery1 failing respectively. From the results we can know that if a key component fails, the system continues to work properly according to the corresponding isolation and reconstruction strategy, namely, bus voltage and motor speed can still maintain stability, which proves redundant design proposed in figure 1 reasonable. Redundant design in energy systems for stratospheric airship plays a important role in long-term reliable flight. 


\section{Conclusions}

Redundancy design and control strategies for stratospheric airship energy management system are proposed in this paper, which can make key node in fault isolated and achieve system reconstruction. Then the simulation results by SIMULINK are shown to verify the theoretical analysis and the control strategies.

\section{Acknowledgements}

This work was supported by the National Natural Science Foundation of China under Grant No. 51307004 and Basic research funds of BeiHang University.

\section{References}

[1] Steve S, Michael F, Michael L: 11th AIAA Aviation Technology, (Turin, Italy, May 8-10, 2011). Vol. 5, p.6973.

[2] Shunichi O, Noboru S, Tsutomu F: 9th Annual International Energy Conversion Engineering Conference (San Diego, California, 31 July - 03 August, 2011). Vol. 2, p.254.

[3] Cui E J: Advances in Mechanics, Vol. 39 (2009) No.6, p.658.

[4] Colozza A: NASA/CR- 2003-212724, 2003.

[5] Michael L, Steve S: 18th AIAA Lighter-Than-Air Systems Technology Conference, (Anchorage, Alaska, March 12-15, 2009). Vol. 2, p.231.

[6] Leng C X, L J and Liu Q F: Transactions of China Electro-technical Society, Vol. 24 (2009) No.4, p.114.

[7] Zhao R F, Ge B M and Bi D Q: Advanced Technology of Electrical Engineering and Energy, Vol. 35 (2009) No.6, p.560.

[8] Sun K W, Zhu M and Xu D D: Applied Mechanics and Materials, Vol. 34 (2014) No.8, p.1765.

[9] Ahmed K, Ahmed M and Shehab A: IEEE Transactions on power electronics, Vol. 26 (2011)

No.9, p.1010.

[10] Qiang M: A Solar Power System for High Altitude Airships (Ph.D., The University of Toledo, China 2011), p.28.

[11] Chen Y A: Practical Simulation and Testing technology of Switch Converter (Mechanical Industry Press, Beijing 2010). p.38.

[12]Venkatesan K: IEEE PESC'89. (New York, May 23-26, 1989). Vol. 5, p.835.

[13] Sun K W, Liang G M and Zhu M. Electrical Technology, Vol. 27 (2012) No.3, p.125.

[14]Qi B J, Du Q and Xu G N: China, 201010115712(2010).

[15] Huang Y: Application research on a stand-alone PV system with ultra-capacitor battery hybrid energy storage (Ph.D., Chongqing University, China 2012), p.48.

[16] Tremblay O, Dessaint L A: World Electric Vehicle Journal, Vol. 12 (2007) No.26, p.284.

[17] Ram K, Yog R S: IEEE Journal of Photovoltaic, Vol. 30 (2013) No.27, p.647.

[18] Somanatham R, Prasad P V: International Symposium on Power Electronics, Electrical Drives, Automation and Motion. (Turin, Italy, July 8-10, 2007).

[19] Guo Z T: H-bridge cascade multi-level inverter circuit and its application (MS., Hebei University of Technology, China 2012). p.30.

[20] Thounthong P, Raël S: Journal of Power Sources, Vol. 193 (2009) No.15, p.376. 